Nervenarzt 2019 9 90:25-34 https://doi.org/10.1007/s00115-018-0602-5 Online publiziert: 5 . September 2018 (c) Der/die Autor(en) 2018

CrossMark

\author{
E. Igde ${ }^{1} \cdot$ A. Heinz $\cdot$ M. Schouler-Ocak ${ }^{2} \cdot$ W. Rössler ${ }^{1,3}$ \\ ${ }^{1}$ Klinik für Psychiatrie und Psychotherapie, Campus Mitte, Charité - Universitätsmedizin Berlin, Berlin, \\ Deutschland \\ ${ }^{2}$ St. Hedwig-Krankenhaus, Tagesklinik Wedding, Psychiatrische Universitätsklinik, Charité, Berlin, \\ Deutschland \\ ${ }^{3}$ Psychiatrische Universitätsklinik Zürich, Zürich, Schweiz
}

\title{
Depressive und somatoforme Störungen bei türkeistämmigen Personen in Deutschland
}

sich die Prävalenz mit zunehmender Aufenthaltsdauer in Deutschland derjenigen der einheimischen Bevölkerung näherte [17].

Es fanden sich insbesondere depressive und somatoforme Störungen bei den türkeistämmigen "Gastarbeitern“ [18]. Aktuelle Untersuchungen berichten bezüglich dieser Störungen im Vergleich erhöhte Prävalenzraten [12, 13]. Die Übersichtsarbeit bezieht sich auf diese häufigen Störungsbilder gemäß Kapitel F3 und F4 in der Internationalen statistischen Klassifikation der Krankheiten und verwandter Gesundheitsprobleme (ICD-10; [5, 13, 20]).

Das Ausmaß depressiver und somatoformer Störungen wird über individuelle Faktoren der persönlichen Verletzlichkeit hinaus auch durch sozioökonomische Faktoren geprägt, so etwa durch das Einkommen, den Bildungsgrad und auf einer gesellschaftlichen Ebene den sozioökonomischen Status der Umgebung, in der man lebt oder aufwächst [15]. Dies wirft die Frage nach relevanten Umweltfaktoren auf, die zur Manifestation depressiver und somatoformer Störungen bei Menschen mit türkischem Migrationshintergrund beitragen, sowie nach ihren Krankheitsvorstellungen und den Bewältigungsmechanismen bezüglich eben dieser Erkrankungen, um die gegebenenfalls erhöhten Prävalenzraten wie auch die Zugangswege zur Gesundheitsversorgung dieser Personengruppe zu verstehen.

\section{Methoden}

Die Literaturrecherche erfolgte online über die MEDLINE-Datenbank (PubMed) sowie über PsycINFO im Zeitraum vom 03. bis 05.07.2018. Es wurden alle Studien eingeschlossen, die zwischen dem 01.01.2000 und dem 05.07.2018 publiziert und in deutscher, englischer oder türkischer Sprache verfasst wurden. Wir gehen davon aus, dass der gewählte Zeitraum die aktuelle Situation dieser Menschen widerspiegelt.

Für die Suche wurde auf PubMed folgende Wortkombination genutzt: mental health OR mental disorder OR mental disease AND Germany AND Turkish. Auf PsycINFO erfolgten drei unabhängige Suchen. Die Suchbegriffe Germany und Turkish waren in allen drei Suchen konstant, es variierten lediglich jeweils die Begriffe mental health, mental disorder und mental disease.

Zusätzlich zu den oben genannten Einschlusskriterien bezüglich des $\mathrm{Pu}$ blikationsdatums und der Sprache der Artikel wurden ausschließlich Originalarbeiten eingeschlossen, die eine definierte Gruppe von Personen mit türkischem Migrationshintergrund in Deutschland beinhalteten, entsprechend der eingangs genannten Definition des Statistischen Bundesamts. Außerdem wurde bei Studien, die manifest psychisch Erkrankte untersuchten, darauf geachtet, dass diese Patienten Diagnosen der Gruppe F3 bzw. F4 laut ICD-10 


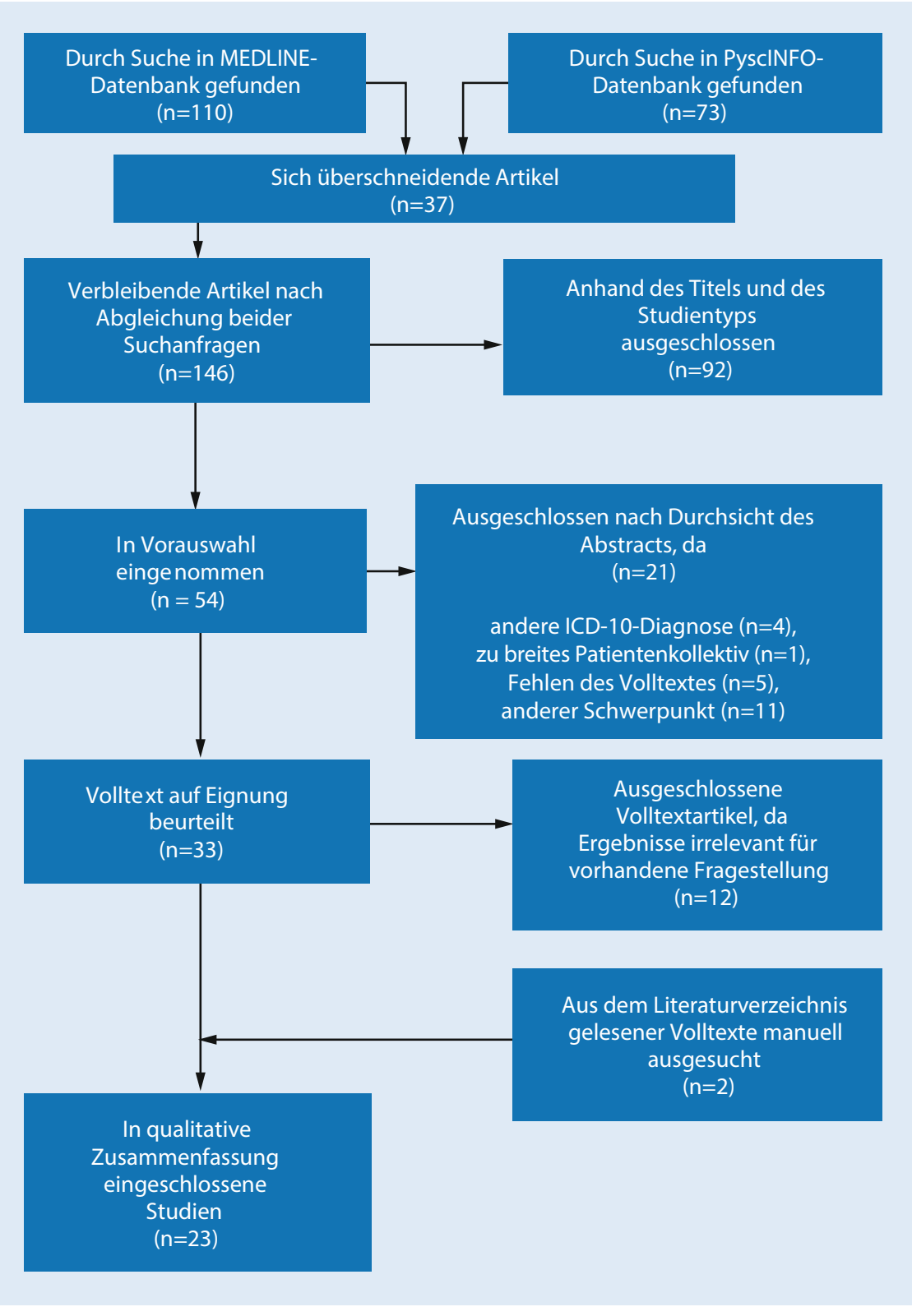

Abb. 1 A Flussdiagramm zur Dokumentation der Literaturrecherche

aufwiesen. Übersichtsarbeiten wurden ausgeschlossen.

In einem ersten Schritt der Vorauswahl wurden Artikel aussortiert, deren Titel bereits erkennen ließ, dass sie nicht relevant für das Thema dieser Arbeit waren. Anschließend wurden weitere Studien nach Lesen der Zusammenfassung ausgeschlossen. Die Volltexte der verbleibenden Studien wurden nachfolgend auf ihre Relevanz für die Fragestellung untersucht.

Um die Aussagekraft der einzelnen Studien zu beurteilen, wurden die Repräsentativität der Populationsstichpro- be sowie der Einschluss potenziell konfundierender Variablen bewertet.

\section{Ergebnisse}

\section{Suchergebnisse}

Die Suche in der MEDLINE-Datenbank ergab 110 Treffer. Auf PsycINFO fanden sich 35 Treffer, wenn der variierende Suchbegriff mental disorder war, 2 Treffer bei dem Begriff mental disease sowie 55 Treffer bei mental health. Innerhalb der Suche in der PsycINFO-Datenbank gab es 19 Überschneidungen, somit lie- ferte diese Suche 73 Ergebnisse. Zunächst wurden beide Suchabfragen abgeglichen. Dabei wurde nach Studien gesucht, die sich in beiden Datenbanken befanden. Es fanden sich 37 Studien sowohl in der MEDLINE- als auch in der PsycINFODatenbank, sodass insgesamt aus beiden Suchen 146 Ergebnisse verblieben. Diese wurden anhand des Titels sowie in weiteren Schritten anhand des Abstracts und des Volltexts auf ihre Eignung überprüft (- Abb. 1). Insgesamt konnten 23 Studien eingeschlossen werden. Alle eingeschlossenen Studien sind Querschnittstudien, es konnten zu dieser Thematik keine Längsschnittstudien gefunden werden.

\section{Ergebnisse der Studienanalyse}

In die Analyse flossen insgesamt die Daten von 19.126 Personen ein. Davon sind 10.507 Frauen und 8403 Männer. Bei 216 Personen gab es keine Angaben zum Geschlecht. In 5 der 23 Studien wurden nur Frauen eingeschlossen. Von den untersuchten 19.126 Personen hatten 9507 einen türkischen Migrationshintergrund bzw. eine türkische Staatsbürgerschaft und 9619 waren deutschstämmig. Hier muss jedoch angemerkt werden, dass die Einteilung in einer Arbeit entsprechend der Staatsbürgerschaft erfolgte, sodass die Gruppe deutscher Staatsbürger eventuell Personen mit türkischem Migrationshintergrund enthielt [23].

Die eingeschlossenen Personen wurden in den analysierten Studien auf unterschiedliche Art und Weise rekrutiert. Unter den Stichproben der 23 untersuchten Studien fanden sich 12 Inanspruchnahmepopulationen, 7 Quotenstichproben, 3 anfallende Stichproben sowie eine Zufallsstichprobe.

Die Hauptergebnisse der einzelnen Studien sind in chronologischer Reihenfolge der Artikel in • Tab. 1 aufgeführt. Die Ergebnisse ermöglichen eine Einteilung in zwei Kategorien, nach denen die weitere Ergebnispräsentation gegliedert ist:

- Soziodemografische und migrationsbedingte Einflussfaktoren

- Individuelle Krankheitsvorstellungen und Einstellung gegenüber Psychotherapie 


\section{Soziodemografische und migra- tionsbedingte Einflussfaktoren}

Bezüglich der soziodemografischen und migrationsbedingten Merkmale weisen die Ergebnisse darauf hin, dass eine erfolgreiche Integration sich protektiv auf das Ausmaß der depressiven Symptomatik auswirkt $[9,29]$. Personen, die als erfolgreich integriert gelten, identifizieren sich sowohl mit der Kultur des Aufnahmelands als auch mit der Kultur des Herkunftslands; sie waren vorwiegend in Deutschland geboren und zweisprachig aufgewachsen [16].

Untersucht man die Lebenszeitprävalenz für psychische Erkrankungen, stellt man jedoch fest, dass keine Unterschiede zwischen türkeistämmigen Personen der ersten und der zweiten Generation bestehen [12]. Diese Gruppen unterscheiden sich jedoch per Definition hinsichtlich ihres Geburtsorts. Zudem bestehen soziodemografische Unterschiede, insbesondere bezüglich des Alters, der Staatsbürgerschaft, der Muttersprache sowie des Bildungs- und sozioökonomischen Status [12]. Die Datenauswertung des Generations and Gender Survey des Bundesinstituts für Bevölkerungsforschung bekräftigt den Einfluss des sozioökonomischen Status, der als eine entscheidende Ursache für den Unterschied im gesundheitlichen Status zwischen Personen mit und ohne Migrationshintergrund dargestellt wird [23].

Aichberger et al. [1] beobachteten einen signifikanten Zusammenhang zwischen dem sozioökonomischen Status und der psychosozialen Belastung bei Patientinnen mit türkischem Migrationshintergrund, wohingegen dieser Zusammenhang bei Patientinnen ohne Migrationshintergrund nicht signifikant war. In beiden Gruppen fand sich jedoch ein signifikanter Zusammenhang zwischen Arbeitslosigkeit und psychosozialer Belastung. Zur Beurteilung des sozioökonomischen Status wurden hier der aktuelle Beschäftigungsstand, der Bildungsstand sowie das Armutsrisiko (berechnet aus dem Nettoeinkommen pro Haushalt) bewertet. Die psychische Belastung wurde mithilfe des General Health Questionnaire 28 (GHQ-28) erfasst.

Nervenarzt 2019 · 90:25-34 https://doi.org/10.1007/s00115-018-0602-5

(c) Der/die Autor(en) 2018

E. Igde · A. Heinz - M. Schouler-Ocak · W. Rössler

\section{Depressive und somatoforme Störungen bei türkeistämmigen Personen in Deutschland}

\section{Zusammenfassung}

Hintergrund. Migration ist ein Risikofaktor für psychische Erkrankungen. Laut epidemiologischen Studien sind die Prävalenzraten depressiver und somatoformer Störungen bei Personen mit türkischem Migrationshintergrund in Deutschland erhöht.

Ziel der Arbeit. Erfassung soziokultureller Einflussfaktoren auf depressive und somatoforme Störungen bei türkeistämmigen Personen in Deutschland.

Material und Methoden. In dieser systematischen Übersicht zu soziokulturellen Faktoren mit Einfluss auf depressive und somatoforme Störungen bei Personen mit türkischem Migrationshintergrund in Deutschland wurden 23 Originalarbeiten aus den Datenbanken PubMed und PsycINFO ausgewertet.

Ergebnisse. Akkulturation, Diskriminierung und sozioökonomischer Status beeinflussen die Schwere der psychischen Belastung und den Zugang zum Gesundheitswesen dieser Personengruppe. Frauen mit türkischem Migrationshintergrund stellen eine besonders vulnerable Gruppe dar.

Diskussion. Die erhöhte Prävalenz depressiver und somatoformer Störungen bei Personen mit türkischem Migrationshintergrund ist multifaktoriell bedingt. Jedoch ist wegen methodischer Mängel der Studien keine Generalisierung auf die gesamte Population Türkeistämmiger in Deutschland möglich. Nationale Erhebungen fehlen weitgehend und es besteht Bedarf an Längsschnittstudien in repräsentativen Kohorten.

\section{Schlüsselwörter}

Türkischer Migrationshintergrund · Epidemiologie · Akkulturation · Sozioökonomischer Status · Diskriminierung

\section{Depressive and somatoform disorders in persons with a Turkish migration background in Germany}

\section{Abstract}

Background. Migration is a risk factor for the onset of mental disorders. Epidemiologic studies indicate that there is an increased prevalence of depressive and somatoform disorders in individuals with a Turkish migration background in Germany. Objectives. The aim of this review article is to determine the impact of sociocultural factors on depressive and somatoform disorders in individuals with a Turkish migration background in Germany.

Materials and methods. The systematic review is based on 23 studies identified in PubMed and PsycINFO.

Results. Acculturation, perceived discrimination and the socioeconomic status affect the severity of mental disorders, and impair the access to the health care system as well as to psychotherapy. Women with a Turkish migration background represent a particularly vulnerable group in this context.

Conclusion. Multiple factors influence the increased prevalence of depressive and somatoform disorders in individuals with a Turkish migration background in Germany. Most of the identified studies suffer from significant methodological restrictions and as such do not allow generalization to the whole population of individuals with a Turkish migration background in Germany. There is a lack of national surveys and a need for longitudinal studies in representative population samples.

\section{Keywords}

Turkish migration - Epidemiology .

Acculturation · Socioeconomic status .

Discrimination
Die psychische Belastung wird bei türkeistämmigen Migrantinnen außerdem durch Erfahrungen sozialer Exklusion und Diskriminierung erhöht [2, 26]. Psychisch Erkrankte mit türkischem Migrationshintergrund berichten dabei häufiger von erlebter Diskriminierung als Personen ohne Migrationshintergrund [30].

Die Studie von Akbiyik et al. [3] verglich die Lebensqualität depressiver $\mathrm{Pa}$ tienten in der Türkei mit der von de- 
Tab. 1 Charakteristika und Ergebnisse der analysierten Studien

\begin{tabular}{|l|l|}
\hline Studie & Kohorte \\
\hline $\begin{array}{l}\text { Calliess et al. } \\
2007\end{array}$ & $\begin{array}{l}\text { 139 türkische Migran- } \\
\text { ten und 164 einhei- } \\
\text { mische Deutsche, } \\
\text { Datenerhebung an } \\
\text { Oberstufenzentren } \\
\text { und Gymnasien }\end{array}$ \\
\hline $\begin{array}{l}\text { Franz et al. } \\
\text { 2007 } \\
{[14]}\end{array}$ & $\begin{array}{l}\text { 79 Patienten tür- } \\
\text { kischer und 79 Pa- } \\
\text { tienten deutscher } \\
\text { Herkunft mit F3- und/ } \\
\text { oder F4-Diagnosen } \\
\text { nach ICD-10 }\end{array}$
\end{tabular}

\begin{tabular}{|c|c|c|c|}
\hline $\begin{array}{l}\text { Akbiyik et al. } \\
2008 \\
\text { [3] } \\
\text { und } 2009 \\
\text { [4] }\end{array}$ & $\begin{array}{l}\text { Patienten mit einer } \\
\text { neu diagnostizierten } \\
\text { Major-Depression, da- } \\
\text { von } 52 \text { Patienten aus } \\
\text { einer psychiatrischen }\end{array}$ & 105 & $\begin{array}{l}\text { Die depressive Symptomatik ist bei den } \\
\text { in Berlin lebenden Patienten höher. Die in } \\
\text { Ankara lebenden Patienten hatten eine hö- } \\
\text { here subjektiv empfundene Lebensqualität } \\
\text { als die in Berlin lebenden Patienten }\end{array}$ \\
\hline
\end{tabular}

Ambulanz in Anka-

ra, 53 Patienten aus

2 psychiatrischen Praxen in Berlin (türkische Psychiater)

Mewes et al. 94 türkische Migran2009

[27]

Gül et al. 2009 [16]

Kotwal 2010 Auswertung der Pati[23]

in Berlin lebenden Patienten höher. Die in Ankara lebenden Patienten hatten eine höals die in Berlin lebenden Patienten

Die türkischen Patientengruppen nannten deutlich mehr somatoforme Symptome als die deutsche Patientengruppe. Der kulturelle Hintergrund war ein relevanter Faktor für die Ausprägung der somatoformen Beschwerden. Bildungsgrad und Berufsstatus hatten ebenfalls einen starken Einfluss auf die Anzahl, die Art und die Ausprägung der angegebenen somatoformen Beschwerden In Gruppe 1 identifizierte sich ein Großteil der Patienten sowohl mit der Türkei als auch mit Deutschland. Sie waren mehrheitlich zweisprachig, hatten die doppelte Staatsbürgerschaft und waren in Deutschland geboren. In Gruppe 2 bezeichneten sich die Patienten größtenteils als Personen mit türkischer Ethnizität, beherrschten die deutsche Sprache weniger gut und waren überwiegend aus der Türkei gebürtig

12.669 Türkische Befragte wiesen eine erhöhte Suszeptibilität für psychische Belastungen ten in Deutschland, 183 in der Türkei lebende Türken sowie 91 einheimische Deutsche, Verteilung der Fragebögen nach dem Schneeballsystem

\section{Patienten mit} türkischem Migrationshintergrund aus psychiatrischer Erstversorgung, davon ordnen sich 154 als gut integriert ein (Gruppe 1) und 66 als marginalisiert (Gruppe 2) entendaten des $\mathrm{Ge}$ nerations and Gender Survey (GGS). 8712 deutsche und 3957 türkische Staatsbürger, die in Deutschland leben

\section{n Ergebnisse}

Deutschstämmige Befragte waren gegenüber einer Psychotherapie positiver eingestellt als die türkischstämmigen Befragten. Gut integrierte Migranten waren besser informiert und aufgeschlossener gegenüber einer Psychotherapie, wohingegen marginalisierte Migranten Stigmatisierungen befürchteten

158 Türkische Patienten nannten mehr körperliche als psychische Symptome und glaubten eher an einen chronischen Verlauf ihrer Erkrankung sowie an negative Konsequenzen. Sie schrieben ihre Erkrankung vorwiegend äußeren Ursachen zu, z. B. der Religion oder den sozioökonomischen Gegebenheiten. Deshalb vermuteten sie häufig, keinen eigenen Einfluss auf den Verlauf ihrer Erkrankung zu haben auf.

Der sozioökonomische Status korrelierte signifikant mit gesundheitlichen Unterschieden.

Türkische Frauen waren in diesem Zusammenhang eine besonders vulnerable Gruppe

\begin{tabular}{|l|l|}
\hline Stichprobe & Limitationen \\
\hline $\begin{array}{l}\text { Anfallende } \\
\text { Stichprobe }\end{array}$ & $\begin{array}{l}\text { Möglicher Bias durch Befra- } \\
\text { gung nichterkrankter Per- } \\
\text { sonen, die noch zur Schule } \\
\text { gehen }\end{array}$ \\
\hline
\end{tabular}

Inanspruchnahmepopulation

Inanspruchnahmepopulation

Anfallende

Stichprobe

Inanspruchnahmepopulation

Zufallsstichprobe

Einzige untersuchte Studie mit national repräsentativer Stichprobe. Jedoch kein Einschluss von eingebürgerten Deutschen, nur Personen mit türkischer Nationalität 
Tab. 1 (Fortsetzung)

\begin{tabular}{|c|c|c|c|}
\hline Studie & Kohorte & $n$ & Ergebnisse \\
\hline $\begin{array}{l}\text { Erim et al. } \\
2011 \\
{[13]}\end{array}$ & $\begin{array}{l}51 \text { Patienten aus } \\
\text { der türkisch-mutter- } \\
\text { sprachlichen Sprech- } \\
\text { stunde einer psy- } \\
\text { chotherapeutischen }\end{array}$ & 51 & $\begin{array}{l}\text { Die türkische Patientengruppe zeigte stär- } \\
\text { ker ausgeprägte depressive Symptome als } \\
\text { die deutsche Referenzgruppe (für die deut- } \\
\text { schen Patienten wurden Referenzwerte des } \\
\text { BDI-Testmanuals genutzt) }\end{array}$ \\
\hline
\end{tabular}

Aichberger

et al. 2012

[1]

405 türkischem Migrationshintergrund, 200 einheimische Deutsche

Bromand et al. 105 türkische Migran2012 tinnen

[10]

Heredia Montesinos et al.

2012

[19]

Vardar et al. 2012

[35]

Balkir et al. 2013

[7]

Sariaslan et al.

2014

[33]

Morawa et al. 2014 [29]
63 türkische Migrantinnen mit einer Depression (F32, F33 oder F34 laut ICD-10) aus psychiatrischen Ambulanzen

70 einheimische Deutsche, 66 Personen mit türkischem Migrationshintergrund in Berlin, 79 Türken in Istanbul

29 türkische Migrantinnen der ersten $\mathrm{Ge}$ neration sowie 27 einheimische Deutsche aus psychiatrischen und psychosomatischen Kliniken mit der Diagnose einer Major-Depression

254 Patienten mit türkischem Migrationshintergrund sowie 164 einheimische deutsche Patienten aus einer allgemeinärztlichen Praxis

254 Patienten aus hausärztlicher Versorgung, 217 Patienten aus ambulanter psychosomatischer Versorgung, beide Gruppen aus türkischsprachigem Behandlungssetting

Stichprobe
Inanspruchnahme-
population

Limitationen

Kleine Kohorte. Vergleich zu den Werten im BDI-Testmanual, dessen Strichprobe eventuell auch Deutsche mit Migrationshintergrund enthält. Patienten ausschließlich aus türkisch-muttersprachlicher Sprechstunde

Quotenstichprobe

Nur Frauen $\rightarrow$ haben im Allgemeinen eine höhere psychische Belastung als Männer

Inanspruchnahmepopulation

Inanspruchnahmepopulation

Kleine Kohorte. Keine Vergleichspopulation vorhanden atik. Die Ausprägung der depressiven Symptomatik, psychische Belastungen und somatische Symptome korrelierten ebenfalls positiv miteinander

Große Heterogenität bezüglich der Erklärungsmodelle zur Definition, Bedeutung, Auslösung und Therapie von psychischen Erkrankungen sowohl zwischen als auch innerhalb der Gruppen. Starker Einfluss des Bildungsniveaus sowie des sozialen Umfelds

56 Die allgemeine psychische Belastung war bei der türkischen Stichprobe höher. Bei den türkischen Migrantinnen zeigte sich zudem eine höhere Ausprägung des interdependenten Selbstkonzepts. Ein solches Konzept ist insbesondere durch Anpassung und Konformität der eigenen Bedürfnisse an die Erwartungen der Bezugsgruppe gekennzeichnet

418 Die türkische Probandengruppe hatte stärkere depressive wie auch somatoforme Symptome als die deutsche Probandengruppe. Die somatoforme Symptomatik war bei Frauen stärker ausgeprägt als bei Männern. Schmerzen stellten bei dem türkischen Patientenkollektiv das vorherrschende somatoforme Symptom dar

471 Eine starke Identifizierung sowohl mit der Kultur des Gastlands als auch mit der des Herkunftslands hatte einen protektiven Effekt auf die depressive Symptomatik
Quotenstichprobe

Inanspruchnahmepopulation

Inanspruchnahmepopulation

Inanspruchnahmepopulation
Studie sehr interessant hinsichtlich der zusätzlichen Gruppe von Personen in Istanbul, jedoch sind auch hier die Stichproben relativ klein

Es wurden ausschließlich Frauen der ersten Generation eingeschlossen. Selbstkonzepte können sowohl vom Geschlecht als auch von der Migrationserfahrung beeinflusst werden

Keine Diagnose einer somatoformen oder depressiven Störung gestellt

Möglicher Bias durch Überrepräsentation von Migranten der ersten Generation. Alle Patienten stammen aus einer türkischsprachigen Sprechstunde 
Tab. 1 (Fortsetzung)

\begin{tabular}{l|l}
\hline Studie & Kohorte \\
\hline $\begin{array}{l}\text { Reich et al. } \\
\text { [32] }\end{array}$ & $\begin{array}{l}\text { 50 türkische Migran- } \\
\text { ten und 50 Deutsche } \\
\text { ohne Migrationshin- } \\
\text { tergrund in stationär } \\
\text { psychiatrischer Be- } \\
\text { handlung mit einer } \\
\text { depressiven, soma- } \\
\text { toformen und/oder } \\
\text { Anpassungsstörung }\end{array}$ \\
\hline $\begin{array}{l}\text { Mewes et al. } \\
2015\end{array}$ & $\begin{array}{l}\text { 214 türkische Mi- } \\
\text { granten und ihre } \\
\text { [26] }\end{array}$ \\
\hline
\end{tabular}

214 Erfahrene Diskriminierung erhöht das Ausmaß der somatoformen und depressiven Symptomatik. Bei Personen, die sich stark mit der diskriminierten Gruppe identifizieren, war dies weniger deutlich zu beobachten

\begin{tabular}{|c|c|}
\hline $\begin{array}{l}\text { Aichberger } \\
\text { et al. } 2015 \\
\text { [2] }\end{array}$ & $\begin{array}{l}205 \text { Patientinnen mit } \\
\text { türkischem Migrati- } \\
\text { onshintergrund }\end{array}$ \\
\hline $\begin{array}{l}\text { Müller et al. } \\
2016 \\
{[30]}\end{array}$ & $\begin{array}{l}62 \text { türkische Patien- } \\
\text { ten (erste oder zwei- } \\
\text { te Generation) und } \\
62 \text { deutsche Patien- } \\
\text { ten, jeweils mit einer } \\
\text { F3- oder F4-Diagnose } \\
\text { nach ICD-10 }\end{array}$ \\
\hline $\begin{array}{l}\text { Brand et al. } \\
2017 \\
{[9]}\end{array}$ & $\begin{array}{l}1126 \text { türkische Mi- } \\
\text { granten und ihre } \\
\text { Nachkommen }\end{array}$ \\
\hline
\end{tabular}

Dingoyan et al. Personen mit türki2017 schem Migrationshin[12] tergrund der ersten $(n=502)$ und der zweiten Generation $(n=151)$

Morawa et al. 335 Personen mit 2017 türkischem Migra[28] tionshintergrund, Rekrutierung über gesellschaftsorientierte Methoden sowie über das Einwohnermeldeamt

Janssen-Kallen- 662 Personen mit berg et al. türkischem Migrati2017 [21] onshintergrund, Interviews auf Türkisch $(n=458)$ oder Deutsch $(n=204)$ gehalten
205 Leibhaftig erlebte Erfahrungen mit ethnischer Diskriminierung erhöhten das Ausmaß der psychischen Belastung

124 In der Patientengruppe mit Migrationshintergrund zeigte sich eine deutlich höhere Prävalenzrate subjektiv wahrgenommener Diskriminierung

1226 Separation und Marginalisierung sind mit einem niedrigeren psychischen Gesundheitsstatus verbunden. Der Akkulturationsstatus kann Unterschiede im Gesundheitsstatus dieser Migrantengruppe erklären

653 Trotz soziodemografischer Unterschiede zwischen der ersten und zweiten Migrationsgeneration, finden sich keine Unterschiede bezüglich der Lebenszeitprävalenz von psychischen Erkrankungen.

Die Lebenszeitprävalenz war bei Frauen, älteren Personen und Personen ohne Partner erhöht

335 Personen mit türkischem Migrationshintergrund hatten stärker ausgeprägte somatoforme Beschwerden als einheimische Deutsche. Eine verstärkte Somatisierung bestand insbesondere bei Frauen mit türkischem Migrationshintergrund und bei Migranten der ersten Generation

662 Es wurde eine sehr hohe 12-Monats-Prävalenz von depressiven Störungen (29\%) erfasst. Ältere Personen sowie Personen mit niedrigem sozioökonomischem Status waren häufiger betroffen. Symptome waren bei Frauen stärker ausgeprägt. Personen, die sich sowohl mit der türkischen als auch mit der deutschen Kultur identifizieren, hatten ein erhöhtes Risiko, an depressiven Störungen zu erkranken (im Widerspruch zu den anderen Studien)
Anfallende

Stichprobe

Stichprobe
Inanspruchnahme-
population

Limitationen

Personen der ersten Generation überrepräsentiert $\rightarrow$ eventuelles Fehlen von Personen mit höherem Bildungsstatus, die eher der zweiten Generation angehören

\section{Quotenstichprobe}

Inanspruchnahmepopulation

Quotenstichprobe

Migranten der ersten Generation sind im Vergleich zur zweiten Generation überrepräsentiert (etwa $80 \%$ zu 20\%)

Quotenstichprobe

Gruppe der Migranten erster Generation ist größer und erwartungsgemäß durchschnittlich deutlich älter

Quotenstichprobe

Symptome zur Somatisierung mittels Selbsteinschätzungsfragebogen ermittelt, vorher keine Diagnose einer somatoformen oder depressiven Erkrankung gestellt

Quotenstichprobe

Mehr als drei Viertel der Befragten sind Migranten der ersten Generation. Die Mehrheit bevorzugte die türkische Sprache für das Interview 
pressiven türkeistämmigen Personen in Deutschland und zeigte, dass die subjektiv empfundene Lebensqualität der in der Türkei lebenden Patienten deutlich höher war als die der in Deutschland lebenden Patienten. In derselben Stichprobe ergab sich eine stärkere depressive Symptomatik bei der Patientengruppe, die in Deutschland lebte [4]. Frauen mit türkischem Migrationshintergrund werden als besonders vulnerable Gruppe hervorgehoben [12, 21, 23].

Die allgemeine psychische Belastung sowie die Depressivität werden zudem vom Umfeld bestimmt: In der Studie von Balkir et al. [7] fand sich in der türkischen Stichprobe ein stärker ausgeprägtes interdependentes Selbstkonzept. Dieses Konzept kennzeichnet sich durch ein hohes Maß an Gruppenzugehörigkeit sowie Anpassung der eigenen Bedürfnisse an die Erwartungen des entsprechenden Umfelds. Patientinnen mit hoch ausgeprägtem interdependentem Selbstkonzept wiesen eine geringere psychische Belastung sowie eine geringere Depressivität auf [7].

\section{Individuelle Krankheitsvorstellun- gen und Einstellung gegenüber Psychotherapie}

Bezüglich der Krankheitswahrnehmung ergab sich, dass subjektive Erklärungsmodelle für psychische Erkrankungen [22] sehr heterogen sind und neben kulturellen Überlieferungen auch durch den Bildungsstand sowie das soziale Umfeld geprägt werden [35].

Bei der Analyse kultureller Faktoren zeigten sich bei türkeistämmigen Personen vorwiegend fatalistisch-externale Krankheitsvorstellungen. Das bedeutet, dass sie ihre Erkrankung häufig aufäußere Ursachen, wie religiöse oder sozioökonomische Faktoren, zurückführten und den eigenen Einfluss auf den Krankheitsverlauf entsprechend gering einschätzten. Dadurch kann die Verantwortung für die Therapie weitgehend auf das professionelle Personal übertragen werden und die Betroffenen verhalten sich bezüglich ihrer eigenen Erkrankung eher passiv. Zudem nannten sie häufiger körperliche Beschwerden, die durch ihre psychiatrische Erkrankung hervorgerufen werden [14].
Diese Beobachtung wurde in weiteren Studien bestätigt: Mewes et al. [27] berichteten von mehr somatoformen $\mathrm{Be}$ schwerden, Erim et al. [13] sowie Sariaslan et al. [33] von einer stärker ausgeprägten depressiven wie auch somatoformen Symptomatik bei türkeistämmigen Personen im Vergleich zu Deutschen ohne Migrationshintergrund. Bei Migranten der ersten Generation war die Somatisierung stärker ausgeprägt als in den nächsten Generationen. Wie bereits bezüglich sozioökonomischer Faktoren erwähnt, stellen Frauen hinsichtlich des Ausprägungsgrads der Somatisierung eine ausgesprochen belastete Gruppe dar [28].

Bezüglich der Aufgeschlossenheit gegenüber einer Psychotherapie fand sich, dass Personen mit türkischem Migrationshintergrund oft weniger motiviert für eine psychotherapeutische Behandlung sind. Allerdings spielt hier der Grad der Integration eine wichtige Rolle: Integrierte türkeistämmige Personen waren bezüglich einer Psychotherapie meist besser informiert und motivierter, wohingegen marginalisierte türkeistämmige Personen weniger aufgeschlossen waren und befürchteten, stigmatisiert $\mathrm{zu}$ werden [11]. Die Angst vor Stigmatisierung korrelierte positiv mit der Ausprägung der depressiven Symptomatik [19]. Laut Reich et al. [32] tragen besonders die fatalistisch-externalen Krankheitsvorstellungen zur fehlenden Motivation für eine Psychotherapie bei.

\section{Diskussion}

Die Qualität der identifizierten Studien unterliegt erheblichen Einschränkungen bezüglich der Repräsentativität der Erhebungen, der Vergleichbarkeit der untersuchten Kollektive und der Erhebungsinstrumente.

\section{Patientenkollektive}

Die Patientenkollektive waren in allen identifizierten Studien mit Ausnahme der Daten des Generations and Gender Survey von Kotwal [23] lokal erhoben. Bei der Studie von Kotwal [23] handelt es sich zudem um die einzige Studie mit einer Zufallsstichprobe und somit um eine repräsentative Population. Größerenteils handelt es sich bei den identifizierten Studien um Inanspruchnahmestudien. Solche Inanspruchnahmestudien umfassen ein hoch selektiertes Klientel und sind nicht repräsentativ für die Gesamtgruppe der türkeistämmigen Personen. Dies gilt auch für die anderen Studiendesigns (anfallende Stichprobe, Quotenstichprobe), wenn auch nicht in dem Ausmaß wie für die Inanspruchnahmepopulation.

Beispielsweise kann das Rekrutieren von Patienten aus ausschließlich türkischsprachigen Sprechstunden [3, 13, 29] bzw. das alleinige Rekrutieren von Personen mit einer türkischen Staatsbürgerschaft [23] dazu führen, dass besser integrierte und sprachlich kompetentere Personen, die möglicherweise nicht nur türkischsprachige Ärzte aufsuchen, sowie Personen, die in Deutschland geboren und aufgewachsen sind, in der Kohorte unterrepräsentiert sind. Da aber genau diese Faktoren das Gesundheitsverhalten bestimmen, wie unsere Analysen gezeigt haben, können wir von unseren Ergebnissen nicht auf die Gesamtpopulation rückschließen. Außerdem bietet nur die Hälfte der genannten Studien eine deutschstämmige Kontrollgruppe.

Bezüglich soziodemografischer Faktoren fanden sich häufig deutliche Unterschiede zwischen den untersuchten Gruppen: So unterschieden sich die Patientenkollektive der türkeistämmigen Personen von denen der einheimischen Deutschen durch einen niedrigeren Bildungsgrad und Berufsstatus sowie durch eine höhere Arbeitslosenquote und ein niedrigeres Einkommen [14, 27, 30]. Zudem wurde in einigen Studien die Ausprägung der depressiven und/oder somatoformen Symptomatik anhand von Fragebögen erfasst, ohne vorher die klinische Diagnose einer solchen Erkrankung gestellt zu haben [28, 33].

\section{Ausprägung depressiver und somatoformer Störungen}

Trotz der Heterogenität und der weitgehend mangelnden Repräsentativität der Stichproben zeigt die Zusammenstellung, dass Menschen mit türkischem Migrationshintergrund stärker ausge- 
prägte depressive und somatoforme Störungen aufweisen [13, 27, 33]. Ein niedriger Bildungs- und sozioökonomischer Status sowie erfahrene Diskriminierung sind mögliche Ursachen für die erhöhte psychische Belastung [1, 23, 26]. Frauen mit türkischem Migrationshintergrund stellen in dem Zusammenhang eine besonders vulnerable Gruppe dar [12, 23]. Der Bildungsstatus hat zusätzlich zu kulturellen Faktoren einen Einfluss auf die Krankheitsvorstellungen, die bei Türkeistämmigen häufig external attribuieren, weshalb diese Personen ihre Krankheit als unkontrollierbar wahrnehmen und $\mathrm{zu}$ einer Psychotherapie wenig motiviert sein können [14]. Integrierte Patienten litten demgegenüber weniger ausgeprägt unter psychischen Erkrankungen und standen einer psychotherapeutischen Behandlung aufgeschlossener gegenüber [11].

\section{Sozioökonomische Faktoren}

Bezüglich der sozioökonomischen Faktoren ist der Zusammenhang eines niedrigen sozialen Status und eines schlechteren Gesundheitsstatus durch zahlreiche epidemiologische Studien gut belegt [24]. Untersuchungen des Bundesamts für Migration und Flüchtlinge zeigen, dass Menschen mit türkischem Migrationshintergrund einen niedrigeren Bildungsstatus, geringere Bildungsabschlüsse und weniger Einkommen haben als andere Personengruppen in Deutschland [36]. Wengler [37] folgert aus der Auswertung der Daten des Generations and Gender Survey, dass die gesundheitlichen Unterschiede zwischen Personen mit türkischem Migrationshintergrund und Deutschen ohne Migrationshintergrund durch die Differenzen im sozioökonomischen Status und den damit verbundenen Bewältigungsstrategien bedingt sind.

In mehreren der analysierten Studien fanden sich dazu passend deutliche Unterschiede zwischen diesen beiden Bevölkerungsgruppen bezüglich des Bildungsniveaus, der Arbeitslosigkeit, des Nettoeinkommens und des allgemeinen sozioökonomischen Status [14, 30]. Zudem stellten wir fest, dass innerhalb des Kollektivs der türkei- stämmigen Personen Unterschiede in der Symptomausprägung [27] und den Krankheitsvorstellungen [14] bestehen, die sich teilweise durch Unterschiede im Bildungs- und sozioökonomischen Status innerhalb dieser Bevölkerungsgruppe erklären lassen [33].

\section{Migrationsbedingte Faktoren}

Mehrere Studien verwiesen auf den Einfluss migrationsbedingter Faktoren wie Akkulturation und erfahrener Diskriminierung, wobei sich Unterschiede zwischen sozial integrierten und marginalisierten Personen zeigten [29]. Eine schlechte Integration sowie schlechte Sprachkenntnisse beeinflussen die Inanspruchnahme des Gesundheitssystems und modifizieren das Gesundheitsverhalten, beginnend damit, dass die Ärzte entsprechend den Sprachkenntnissen gewählt werden. Diese Faktoren hängen zudem auch voneinander ab: Gute Kenntnisse der deutschen Sprache finden sich häufiger in der integrierten sowie in der assimilierten Gruppe [9]. Integrierte Personen haben außerdem im Vergleich zu marginalisierten Personen zwei Systeme sozialer Unterstützung: sowohl ihre ethnische Gruppe als auch die Gastgesellschaft [29], was bezüglich der Krankheitsbewältigung von Bedeutung sein kann.

Unterschiedliche Erklärungsmodelle psychischer Erkrankungen können zu Missverständnissen zwischen deutschstämmigen Behandlern und Patienten mit türkischem Migrationshintergrund führen, die die Nutzung des Gesundheitssystems beeinträchtigen [31]. Die Studie von Vardar et al. [35] warnt allerdings vor Pauschalisierungen und weist darauf hin, dass die Krankheitsmodelle von Menschen mit türkischem Migrationshintergrund in Abhängigkeit vom Bildungsgrad und anderen Faktoren genauso heterogen ausfallen wie die der Menschen ohne Migrationshintergrund, sodass die individuelle Anamnese nicht durch generelle Kenntnisse kultureller Traditionen ersetzt werden kann.

Individuelle Unterschiede im Verständnis, wie psychische Störungen entstehen, aufrechterhalten und behandelt werden, werden durch unterschiedliche
Coping-Strategien im Umgang mit der psychischen Erkrankung ergänzt, die durch Ressourcen des sozialen Umfelds ebenso geprägt werden wie durch das individuelle Selbstwertgefühl oder das Ausmaß verschiedener Persönlichkeitsfaktoren einschließlich der Extraversion [10].

\section{Limitationen}

Die Hauptlimitation der vorliegenden systematischen Übersicht besteht in der mangelnden Repräsentativität der analysierten Studien. Mit einer Ausnahme [23] machen die Größe der Kohorten sowie die oft unsystematische Rekrutierung das Studiendesign anfällig für Konfundierungen und fehlerhafte Interpretationen. Eine weitere Limitation besteht darin, dass nur die Datenbanken PubMed und PsycINFO zur Literaturrecherche genutzt wurden, allerdings wurden alle weiteren einschlägigen Studien, die sich im Verlauf der Untersuchung in den gefundenen Artikeln fanden, ergänzend einbezogen.

Kritisch anzumerken ist die Heterogenität der Patientenkollektive der untersuchten Studien. Diese bestehen manchmal nur aus Personen mit einer türkischen Staatsbürgerschaft [30], in einigen Studien aus Menschen mit türkischem Migrationshintergrund gemäß der eingangs genannten Definition [2, 6, 16] und in anderen aus Menschen der ersten und/oder zweiten Einwanderergeneration $[11,19]$.

Personen der ersten Migrationsgeneration sind in der Türkei geboren, haben häufig bessere Türkisch- als Deutschkenntnisse und identifizieren sich mehr mit der Kultur des Herkunftslands als mit der des Gastlands [29]. Aus diesen Gründen würden wir bei dieser Gruppe eine stärkere Symptombelastung bei depressiven und somatoformen Störungen erwarten, da die Analyse gezeigt hat, dass die oben genannten Faktoren in diesem Zusammenhang eine Rolle spielen [28, 29]. Das erschwert deutlich die Interpretation der Studien, in denen die Kollektive Personen mehrerer Migrationsgenerationen enthalten, da diese sich bezüglich entscheidender Faktoren unterscheiden, die sowohl die Krankheitsvorstellungen 
als auch die Entstehung und den Umgang mit ihrer Erkrankung zu beeinflussen vermögen.

Die eigene Migrationserfahrung, Deutschkenntnisse, der Akkulturationsgrad sowie der sozioökonomische Status variieren somit entsprechend dem Patientenkollektiv sowie innerhalb der Patientenkollektive und erschweren es, einzelne Faktoren zu identifizieren, die bei türkeistämmigen Personen die Unterschiede im Bereich der psychischen Gesundheit erklären. Zudem sind fast alle Personengruppen in Großstädten (Berlin, Essen, Hamburg und Marburg) rekrutiert worden, sodass Studien im ländlichen Gebiet weitgehend fehlen.

Die Erhebung der Daten erfolgte in allen Studien mit standardisierten Fragebögen, die sich jedoch je nach Zielsetzung unterschieden und somit verschiedene Fragen und Skalen beinhalteten. Da der Fokus dieser Übersichtsarbeit somatoformen und depressiven Störungen galt, wurden Studien, die sich auf andere psychiatrische Störungen beziehen, nicht einbezogen.

\section{Resümee}

Zusammenfassend bieten die analysierten Studien trotz aller Limitationen wichtige Erkenntnisse zur psychischen Gesundheit von Personen mit türkischem Migrationshintergrund in Deutschland. Weitgehend übereinstimmend zeigte sich eine erhöhte Prävalenz depressiver und somatoformer Störungen, [12, 34]. Besonders stark ausgeprägt war sie bei vulnerablen Gruppen wie Frauen [28] und/ oder Personen mit relativ geringen psychosozialen Ressourcen [1] und verstärkter Exposition gegenüber sozialer Diskriminierung und Exklusion [2, 26, 32]. Die Gruppe der Menschen mit türkischem Migrationshintergrund ist heterogen, auch bezüglich ihrer Erklärungsmodelle psychischer Krankheit [35], die nicht pauschal voraussetzbar sind, sondern im individuellen therapeutischen Kontext erfragt werden müssen. Nationale Erhebungen fehlen weitgehend. Es besteht Bedarf an Längsschnittstudien mit repräsentativen Kohorten.

\section{Fazit für die Praxis}

- Die erhöhte Prävalenz depressiver und somatoformer Störungen bei Personen mit türkischem Migrationshintergrund ist multifaktoriell bedingt.

- Zu diesen Faktoren gehören insbesondere der sozioökonomische und der Bildungsstatus sowie soziokulturelle Faktoren, psychosoziale Ressourcen und erfahrene Diskriminierung.

- Frauen mit türkischem Migrationshintergrund stellen eine besonders vulnerable Gruppe dar.

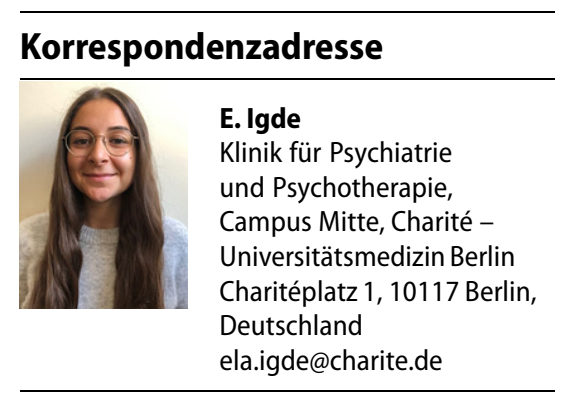

Einhaltung ethischer Richtlinien

Interessenkonflikt. E. Igde, A. Heinz, M. SchoulerOcak und W. Rössler geben an, dass kein Interessenkonflikt besteht.

Dieser Beitrag beinhaltet keine von den Autoren durchgeführten Studien an Menschen oder Tieren.

Open Access Dieser Artikel wird unter der Creative Commons Namensnennung 4.0 International Lizenz (http://creativecommons.org/licenses/by/4.0/deed. de) veröffentlicht, welche die Nutzung, Vervielfältigung, Bearbeitung, Verbreitung und Wiedergabe in jeglichem Medium und Format erlaubt, sofern Sie den/die ursprünglichen Autor(en) und die Quelle ordnungsgemäßnennen, einen Linkzur Creative Commons Lizenz beifügen und angeben, ob Änderungen vorgenommen wurden.

\section{Literatur}

1. Aichberger MC, Bromand Z, Heredia Montesinos A et al (2012) Socio-economic status and emotional distress of female Turkish immigrants and native German women living in Berlin. Eur Psychiatry 27(Suppl2):S10-S16

2. Aichberger MC, Bromand Z, Rapp MA et al (2015) Perceived ethnic discrimination, acculturation, and psychological distress in women of Turkish origin in Germany. Soc Psychiatry Psychiatr Epidemiol 50:1691-1700

3. Akbiyik DI, Berksun OE, Sumbuloglu V et al (2008) Quality of life of Turkish patients with depression
Hier steht eine Anzeige. Springer 
in Ankara and in Berlin. Eur Psychiatry 23(Suppl 1):4-9

4. Akbiyik DI, Sumbuloglu V, Berksun OE et al (2009) Symptoms of Turkish patients with depression in Ankara and Berlin. Cult Med Psychiatry 33:412-420

5. ICD-10-WHO-Version 2016. Deutsches Institut für Medizinische Dokumentation und Information (DIMDI). http://www.dimdi.de/static/de/ klassi/icd-10-who/kodesuche/onlinefassungen/ htmlamtl2016/index.htm. Zugegriffen: 10. Dez. 2017

6. Statistisches Bundesamt (2017) Bevölkerung mit Migrationshintergrund - Ergebnisse des Mikrozensus, Zahlen und Fakten zu Migration und Integration. https://www.destatis.de/DE/ ZahlenFakten/GesellschaftStaat/Bevoelkerung/ MigrationIntegration/Migrationshintergrund/ Tabellen/MigrationshintergrundStaatsange hoerigkeitStaaten.html.Zugegriffen: 17.Dez. 2017

7. Balkir N, Arens EA, Wolff C et al (2013) The link between self-construal and mental distress in Turkish migrants and German women with depression. Psychiatr Prax 40:135-141

8. Bermejol,MayningerE, Kriston Letal (2010) Mental disorders in people with migration background compared with German general population. Psychiatr Prax 37:225-232

9. Brand T, Samkange-Zeeb F, Ellert U et al (2017) Acculturation and health-related quality of life: results from the German National Cohort migrant feasibility study. Int J Public Health 62:521-529

10. Bromand Z, Temur-Erman S, Yesil R et al (2012) Mental health of Turkish women in Germany: resilience and risk factors. Eur Psychiatry 27(Suppl 2):S17-S21

11. Calliess IT, Schmid-Ott G, Akguel G et al (2007) Attitudes towards psychotherapy of young second-generation Turkish immigrants living in Germany. PsychiatrPrax 34:343-348

12. Dingoyan D, Schulz $H$, Kluge $U$ et al (2017) Lifetime prevalence of mental disorders among first and second generation individuals with Turkish migration backgrounds in Germany. BMC Psychiatry 17:177

13. Erim Y, Morawa E, Ozdemir DF et al (2011) Prevalence, comorbidity and severity of psychosomatic disorders in outpatients with Turkish migration background. Psychother Psychosom Med Psychol 61:472-480

14. Franz M, Lujic C, Koch E et al (2007) Subjective illness beliefs of Turkish migrants with mental disorders - specific characteristics compared to german patients. Psychiatr Prax 34:332-338

15. Gruebner O, Rapp MA, Adli Met al (2017) Cities and mental health. Dtsch Arztebl Int 114:121-127

16. Gul V, Kolb S (2009) Acculturation, bicultural identity and psychiatric morbidity in young Turkish patients in Germany. Turk Psikiyatri Derg 20:138-143

17. Hafner H (1980) Psychiatric morbidity among immigrant workers in Mannheim (author's transl). Nervenarzt 51:672-683

18. Hafner H, Moschel G, Ozek M (1977) Psychiatric disturbances in Turkish guest-workers in Germany. A prospective study of 200 immigrants (author's transl). Nervenarzt 48:268-275

19. Heredia Montesinos A, Rapp MA, Temur-Erman S et al (2012) The influence of stigma on depression, overall psychological distress, and somatization among female Turkish migrants. Eur Psychiatry 27(Suppl 2):S22-S26

20. Jacobi F, Hofler M, Siegert J et al (2014) Twelvemonth prevalence, comorbidity and correlates of mental disorders in Germany: the Mental Health
Module of the German Health Interview and Examination Survey for Adults (DEGS1-MH). Int J Methods Psychiatr Res 23:304-319

21. Janssen-Kallenberg $H$, Schulz $H$, Kluge $U$ et al (2017) Acculturation and other risk factors of depressive disorders in individuals with Turkish migration backgrounds. BMC Psychiatry 17:264

22. Kleinman A (1980) Patients and healers in the context of culture: an exploration of the borderland between anthropology, medicine, and psychiatry. University of California Press, Berkeley

23. Kotwal AA (2010) Physical and psychological health of first and second generation Turkish immigrantsin Germany. Am JHum Biol 22:538-545

24. Lampert T, Richter M, Schneider S et al (2016) Social inequality and health: Status and prospects of socio-epidemiological research in Germany. Bundesgesundheitsblatt Gesundheitsforschung Gesundheitsschutz 59:153-165

25. Lindert J, Schouler-Ocak M, Heinz A et al (2008) Mental health, health care utilisation of migrants in Europe. Eur Psychiatry 23(Suppl 1):14-20

26. Mewes R, Asbrock F, Laskawi J (2015) Perceived discrimination and impaired mental health in Turkish immigrants and their descendents in Germany. Compr Psychiatry 62:42-50

27. Mewes R, Rief W (2009) Are somatoform complaints and causal attributions in Turkish migrants associated with their cultural background or the migration itself? Z Med Psychol 18:135

28. Morawa E, Dragano N, Jockel KH et al (2017) Somatization among persons with Turkish origin: results of the pretest of the German national cohort study. JPsychosom Res 96:1-9

29. Morawa E, Erim Y (2014) Acculturation and depressive symptoms among Turkish immigrants in Germany. Int J Environ Res Public Health 11:9503-9521

30. Muller MJ, Koch E (2016) Perceived discrimination in patients with psychiatric disorder and Turkish migration background in Germany. JNerv Ment Dis 204:542-546

31. Penka S, Faisst $H$, Vardar A et al (2015) The current state of intercultural opening in psychosocial services-the results of an assessment in an innercity district of Berlin. Psychother Psychosom Med Psychol 65:353-362

32. Reich H, Bockel L, Mewes R (2015) Motivation for psychotherapy and illness beliefs in Turkish immigrant inpatients in Germany: results of a cultural comparison study. J Racial Ethn Health Disparities 2:112-123

33. Sariaslan S, Morawa E, Erim Y (2014) Mental distress in primary care patients: German patients compared with patients of Turkish origin. Nervenarzt 85:589-595

34. Tydecks S, Temur-Erman S, Schouler-Ocak M et al (2009) Mental disorders, mental distress and social support among a sample of Turkish immigrants in two general practitioners' offices in Berlin. Z Med Psychol 18:101-107

35. Vardar A, Kluge U, Penka S (2012) How to express mental health problems: Turkish immigrants in Berlin compared to native Germans in Berlin and Turks in Istanbul. Eur Psychiatry 27(Suppl 2):S50-S55

36. Von Gostomski C (2010) Fortschritte der Integration: Zur Situation der fünf größten in Deutschland lebenden Ausländergruppen. Bundesamt für Migration und Flüchtlinge (BAMF), Berlin

37. Wengler A (2011) The health status of firstand second-generation Turkish immigrants in Germany. Int J Public Health 56:493-501
Genetik von Intelligenz und kognitiven Störungen

Ein komplexes, aber relevantes Thema - nicht nur für die Humangenetik

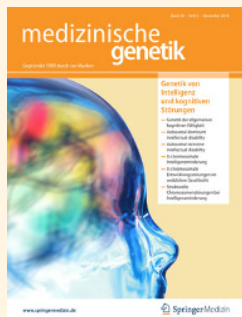

Intelligenz ist eines der bestuntersuchten Konstrukte der empirischen Verhaltenswissenschaften. Während der letzten Jahre haben die neuen Hochdurchsatz-Sequenzierungsverfahren zur Identifizierung von zahlreichen neuen Genen für die autosomal-dominante, autosomal-rezessive und X-gebundene Intelligenzminderung geführt.

Lesen Sie in Heft 3/2018 (November) der Zeitschrift medizinische genetik über die aktuellen Kenntnisse der Wissenschaft zum Thema Genetik von Intelligenz und kognitiven Störungen.

- Genetik der allgemeinen kognitiven Fähigkeit

- Autosomal dominante geistige Behinderung

- Autosomal rezessive geistige Behinderung

- X-chromosomale geistige Behinderung bei Jungen

- X-chromosomale geistige Behinderung bei Mädchen

- Strukturelle

Chromosomenaberrationen bei Intelligenzminderung

OPEN ACCESS - Diese Beiträge sind für Sie online frei verfügbar!

Suchen Sie noch mehr zum Thema? Mit e.Med - den maßgeschneiderten Fortbildungsabos von Springer Medizin - haben Sie Zugriff auf alle Inhalte von SpringerMedizin.de. Sie können schnell und komfortabel in den für Sie relevanten Zeitschriften recherchieren und auf alle Inhalte im Volltext zugreifen.

Weitere Infos zu e.Med finden Sie auf springermedizin.de unter „Abos" 